\title{
SENILE ANOREXIA AND HYPERALDOSTERONISM
}

\author{
D. Schwerthöffer, T. Grimmer, I. Bauer
}

\begin{abstract}
Objective and Method: A case presentation of a 73-year-old delirious patient diagnosed with hyperaldosteronism and Anorexia Nervosa. A possible pathophysiological relationship between a decades-long uncontrolled eating disorder (so called Senile Anorexia) and a resulting transformation of the adrenal gland is discussed. Results and Conclusion: Senile anorexia can remain undetected for decades only to then manifest itself through somatic complications, e.g. symptoms of hyperaldosteronism. In older, underweight patients with unclear neuropsychiatric symptoms, the diagnosis of Anorexia Nervosa should be considered.
\end{abstract}

Key words: Senile anorexia, hyperaldosteronism, anorexia nevrosa.

\section{Introduction}

Patients with senile anorexia diagnosed decades after the onset of the disease usually exhibit a multitude of anorexia-related somatic comorbidities (1). This complicates both the diagnosis of the underlying eating disorder as well as the differential diagnosis of occurring somatic symptoms.

We present the case of a 73-year-old delirious patient diagnosed with hyperaldosteronism and discuss the possible pathophysiological relationship between a decades-long uncontrolled eating disorder and resulting adrenal gland changes.

\section{Patient}

\section{History of Present Illness}

A 73-year-old patient was hospitalized for psychiatric treatment for the first time ever due to cognitive deficits that had first appeared two years prior. At the time of admission she was short-tempered, showed attentional deficits and poor concentration as well as impaired orientation to time, place, and situation. She received 19 of 30 points on the Mini-Mental State Examination (MMSE). The patient's husband reported his wife having exhibited disorganised behaviour, having become increasingly disoriented, and having fallen repeatedly within the four preceding weeks.

Klinik und Poliklinik für Psychiatrie und Psychotherapie der TU-München, Germany

Corresponding Author: Dirk Schwerthöffer, Klinik für Psychiatrie und Psychotherapie der TU-München, Germany, Dirk.Schwerthoeffer@mri.tum.de

Received September 6, 2017

Accepted for publication November 6, 2017

\section{Past Medical History}

The patient had a history of hypertension and multiple contusions of the head, forehead, and extremities secondary to falls at home. Her general practitioner had been prescribing her oral potassium for several years to correct hypokalaemia.

Low body weight with a body mass index (BMI) of 13 were noted upon physical examination.

\section{Indirect Anamnesis}

Her family reported that the patient had always exhibited eating habits that did not conform to social norms. She ate irregularly, preferring to eat alone and never with family. The patient later admitted to having self-induced vomiting for years.

\section{Neuropsychological Assessment}

Distinct cognitive deficits (MMSE 19/30) and massive impairment of executive function and cognitive flexibility, among other findings, were noted, raising suspicion of dementia or a dementia-mimicking process.

\section{Laboratory Findings (Serum)}

Hyponatraemia was discovered $123 \mathrm{mmol} / \mathrm{l}$ (normal >135). Potassium was normal due to oral substitution. Aldosterone was within physiological limits (80.8 pg/ $\mathrm{ml})$, elevated aldosterone-to-renin ratio of $115 \mathrm{pg} / \mathrm{ml}: \mu \mathrm{l}$ (normal $<12$ ). 


\section{Diagnostic Procedures}

Electrocardiography (ECG) and electroencephalography (EEG) showed no pathological findings. Cranial magnetic resonance imaging (cMRI) uncovered slight microangiopathic changes without a specific pattern of atrophy. Magnetic resonance imaging (MRI) of the adrenal gland showed enlargement of the left adrenal gland, leading to the suspicion of an adrenal adenoma (Figure 1).

\section{Conclusion}

The elevated aldsMRI scan suggest primary hyperaldosteronism.

\section{Figure 1}

Abdominal MRI scan, axial plane, T1-weighted image

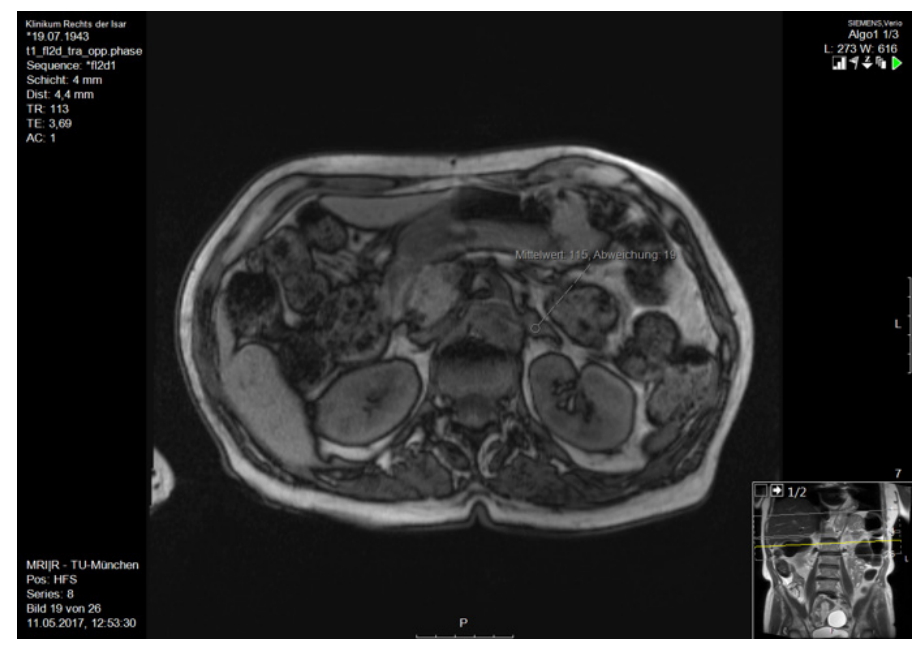

Finding: enlargement of the left adrenal gland suggestive of an adrenal adenom

\section{Course of Treatment}

Under oral substitution of sodium, serum sodium normalized and the patient's psychopathological status improved. After one week she was fully orientated, no longer irritable, and the cognitive deficits with which she had presented improved. The aldosterone antagonist spironolactone $(25 \mathrm{mg})$ was administered owing to her diagnosis of 'hyperaldosteronism with electrolyte disturbances'. Her MMSE score reached 28/30 at the time of discharge.

The patient received further endocrinologic treatment because of the suspected adrenal adenoma (differential diagnosis hyperplasia). A positron emission tomography scan (CXCR4-PET) was planned in order to exclude a multiple endocrine neoplasia (MEN).

\section{Summary and Diagnostic Considerations}

At the time of admission, the patient suffered from delirium due to hyponatraemia. After electrolyte normalization, the delirious state subsided. The electrolyte disturbance was caused by self-induced vomiting and hyperaldosteronism. Due to the patient's history and low body weight, a decades-long uncontrolled anorexia nervosa must be assumed.

This raises the question of whether there is a relationship between the patient's anorectic eating disorder and her hyperaldosteronism and whether the electrolyte imbalance was the cause or consequence of hyperaldosteronism.

\section{Hyperaldosteronism}

\section{Primary Hyperaldosteronism}

Primary hyperaldosteronism (Conn's syndrome) is the result of excess production of aldosterone by the zona glomerulosa of the adrenal cortex (2). Common symptoms include electrolyte disturbances (especially hypokalaemia) and hypertension. Approximately $70 \%$ of cases are due to bilateral adrenal hyperplasia, 30\% to an aldosterone-producing adrenal adenoma, and in rare instances a familial variant is the cause (3).

\section{Secondary Hyperaldosteronism}

Secondary hyperaldosteronism is defined as elevated aldosterone production due to extrapituitary, extraadrenal stimulation of the adrenal gland. Frequent causes are renal artery stenosis or hypovolaemia. In both, reduced blood supply across the juxtaglomerular apparatus stimulates the renin-angiotensin-aldosterone system, leading to increased aldosterone release. (4).

\section{Senile Anorexia}

Declining appetite in old age can have numerous physiological (e.g. homeostasis of appetite-regulating hormones, reduced nutritional needs through declining muscle mass, declining olfactory sense or sense of taste) and pathophysiological (e.g. physical illness, depression, cytokine-mediated anorexia-cachexia syndrome, medication intake) causes (5-7). The question whether anorexia nervosa can first manifest itself in old age was controversially discussed in the past $(8,9)$. Despite sporadic reports suggesting this possibility (10), the onset of anorexia in elderly patients is largely assumed to have occurred in early puberty yet escaped early diagnosis (8).

Since widely varying degrees of eating behaviour (from slightly reduced nutritional intake to lifethreateningly reduced nutritional intake) and course of the disease (episodic, regularly intermittent, chronic) 
Table 1

Similarities and differences of senile, juvenile and physiologic senile anorexia

\begin{tabular}{llll}
\hline & $\begin{array}{l}\text { Late diagnosed anorexia } \\
\text { (Senile anorexia) }\end{array}$ & $\begin{array}{l}\text { Early diagnosed anorexia } \\
\text { (Juvenile anorexia) }\end{array}$ & Physiologic senile anorexia \\
\hline Sex & Mostly female & Mostly female & Both sexes \\
First manifestation & Youth/puberty & Youth/puberty & Old age \\
Somatic comorbidities & Late, numerous & Late, numerous & Late, slowly developing \\
Psychic comorbidities & Numerous & Numerous & Rarely (mostly depression) \\
Hospital stays & Late & Early & Late, less often \\
Partnership & Yes, long-lasting, stable-symbiotic & No, infrequent, short & Appears more often in singles \\
Illness insight & Little & Little & Sometimes \\
\hline
\end{tabular}

exist, one can speak of a continuum of anorectic eating disorders with fluid boundaries. Patients with anorexia nervosa diagnosed late represent a distinct subgroup along this continuum and can exhibit common psychopathological and psychosocial characteristics (Tab.1). Apart from medical influences (brain organic and hormonal), psychological, social and cultural factors for the development and course of anorexia nervosa are being discussed (11). A stable, at times symbiotic partnership could positively influence the course of the disease for patients with anorexia nervosa, postponing the need for diagnosis and treatment.

The frequency of senile anorexia could be on the rise due in part to the health and slimness movements of the 70 s and 80s, which legitimized and praised unhealthy body standards. It is possible that there is a large number of undiagnosed cases, which may only be detected in old age through keen investigation.

\section{Hyperaldosteronism in Senile Anorexia}

Patients with senile anorexia suffer from a multitude of somatic and psychiatric comorbidities. In addition to the well-known somatic consequences of anorexia nervosa, less common secondary diseases like hyperaldosteronism can occur. Lifelong electrolyte imbalances (especially hyponatraemia) could lead to hyperplasia of the zona glomerulosa of the adrenal cortex through continuous stimulation of the renin-angiotensin-aldosterone system. Thus, a vicious circle such as in Figure 2 could arise.

\section{Discussion}

\section{Connection between Electrolyte Imbalance, Hyperaldosteronism and Anorexia}

In the presented case, the initial symptoms of delirium and the pre-existing cognitive deficits could be attributed to electrolyte imbalance due to longtime self-induced vomiting and hyperaldosteronism. Adrenal hyperplasia may be the result of continuous stimulation of the reninangiotensin-aldosterone system (Fig. 2). The falls at home the patient suffered prior to inpatient treatment could have been a consequence of hypokaelemia-induced muscular weakness (12). Whether the left adrenal gland enlargement was the result of an adenoma could not be determined by the time of publication. The patient is currently receiving further testing and treatment. The unbalanced nature of an adenoma coupled with anorexia nervosa could have led to life-threatening organic disease. Ultimately, it is impossible in this case to distinguish between primary and secondary hyperaldosteronism because secondary hyperaldosteronism could have resulted in a hyperplastic adenoma.

\section{Figure 2}

Anorexia, hyperaldosteronism and cognitive deficits possible pathomechanism

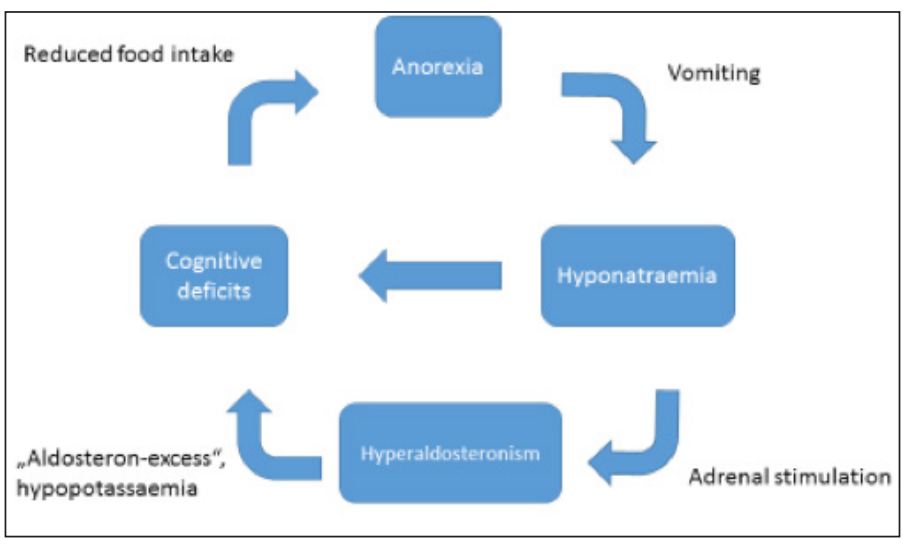

Neuropsychiatric Symptoms in Hyperaldosteronism

Numerous endocrine disorders, e.g. hypercortisolism, hyperthyroidism, hypothyroidism, and hyperparathyroidism (13), can cause neuropsychiatric symptoms (14). Hyperaldosteronism is known to cause anxiety disorders and symptoms of depression 
(15). The exact pathophysiologic link is unknown but could develop as shown in Figure 2 and detailed in the presented case.

\section{Senile Anorexia}

Senile anorexia can remain undetected for decades only to then manifest itself through somatic, e.g. endocrine symptoms (1). A higher age at first admission for the treatment of anorexia nervosa predicts a negative course of the illness (16).

\section{Conclusion}

In older, underweight patients with unclear neuropsychiatric symptoms, the diagnosis of anorexia nervosa with concomitant endocrine and pathophysiologic alterations should be considered.

\section{References}

1. Schwerthoffer D, Fatke B, Bauml J, Forstl H. Senile anorexia: three cases with complicated treatment and two occurrences of a Pisa syndrome. International journal of geriatric psychiatry. 2012;27(7):765-8.

2. Gyamlani G, Headley CM, Naseer A, Valaulikar GS, Geraci SA. Primary Aldosteronism: Diagnosis and Management. The American journal of the medical sciences. 2016;352(4):391-8.

3. Schirpenbach C, Segmiller F, Diederich S, Hahner S, Lorenz R, Rump LC, et al. The diagnosis and treatment of primary hyperaldosteronism in Germany: results on 555 patients from the German Conn Registry. Deutsches Arzteblatt international. 2009;106(18):305-11.
4. Stowasser M, Gordon RD. Primary Aldosteronism: Changing Definitions and New Concepts of Physiology and Pathophysiology Both Inside and Outside the Kidney. Physiological reviews. 2016;96(4):1327-84.

5. Thomas DR. Anorexia: aetiology, epidemiology and management in older people. Drugs \& aging. 2009;26(7):557-70.

6. Volkert D, Frauenrath C, Oster P, Schlierf G. [Malnutrition in the aged-effect of physical, mental, psychological and social factors]. Zeitschrift fur Gerontologie. 1989;22(1):6-10.

7. Donini LM, Savina C, Piredda M, Cucinotta D, Fiorito A, Inelmen EM, et al. Senile anorexia in acute-ward and rehabilitations settings. The journal of nutrition, health \& aging. 2008;12(8):511-7.

8. Scholtz S, Hill LS, Lacey H. Eating disorders in older women: does late onset anorexia nervosa exist? The International journal of eating disorders. 2010;43(5):393-7.

9. Lapid MI, Prom MC, Burton MC, McAlpine DE, Sutor B, Rummans TA. Eating disorders in the elderly. International psychogeriatrics. 2010;22(4):52336.

10. Beck D, Casper R, Andersen A. Truly late onset of eating disorders: a study of 11 cases averaging 60 years of age at presentation. The International journal of eating disorders. 1996;20(4):389-95.

11. Miller CA, Golden NH. An introduction to eating disorders: clinical presentation, epidemiology, and prognosis. Nutrition in clinical practice : official publication of the American Society for Parenteral and Enteral Nutrition. 2010;25(2):110-5

12. Etgen T, Grabert C. [Tetraparesis with hypertensive crisis : hypokalemic rhabdomyolysis in primary hyperaldosteronism]. Der Nervenarzt. 2009;80(6):717-9.

13. Paslakis G, Gilles M, Frankhauser P, Lanczik O, Deuschle M, Frolich L, et al Two cases of primary hyperparathyroidism with depressive and cognitive symptoms. The journal of nutrition, health \& aging. 2010;14(9):798-9.

14. Kunzel HE. Psychopathological symptoms in patients with primary hyperaldosteronism--possible pathways. Hormone and metabolic research $=$ Hormon- und Stoffwechselforschung $=$ Hormones et metabolisme . 2012;44(3):202-7.

15. Sonino N, Tomba E, Genesia ML, Bertello C, Mulatero P, Veglio F, et al. Psychological assessment of primary aldosteronism: a controlled study. The Journal of clinical endocrinology and metabolism. 2011;96(6):E878-83.

16. Fichter MM, Quadflieg N, Crosby RD, Koch S. Long-term outcome of anorexia nervosa: Results from a large clinical longitudinal study. The International journal of eating disorders. 2017. 